\title{
Modelling and Control of a Complementary Energy Recuperation System for Mobile Working Machines
}

\author{
A. Hugo , K. Pettersson, K. Heybroek*, and P. Krus \\ Division of Fluid and Mechatronic Systems, Department of Management and Engineering, Linköping University, Sweden \\ E-mail: anton_hugo@hotmail.com, karl.pettersson@liu.se, kim.heybroek@volvo.com, petter.krus@liu.se \\ "Volvo Construction Equipment AB, SE-631 85 Eskilstuna, Sweden
}

\begin{abstract}
The concept of hybrid technologies for mobile working machines has gained increased attention in recent years. This paper deals with a parallel hybrid system for energy recuperation based on a two-machine hydraulic transformer. The system can be connected hydraulically to an existing hydraulic circuit as a complementary add-on system. The linear analysis of the system visualises the control difficulties coming from a low inertia, slow control dynamics of the machines and the non-linear stick-slip friction during low speeds. A control strategy based on linear control methods is proposed and evaluated in a hardware test bench. It is shown that an acceptable performance can be achieved even with fairly simple models. Additionally, a start-up procedure is proposed to start the transformer from zero speed.
\end{abstract}

Keywords: Parallel hybrid, Energy recuperation, Hydraulic transformer

\section{Introduction}

If hydraulic power would let itself be transformed from one pressure level to another as easy, cheap and efficient as electric power does, a great roadblock for the future of hydraulics would be out of the way. Within electrics the transistor technology has revolutionised the switching transformers in terms of size, efficiency and cost. In hydraulics, the digital approach is still in its cradle and so far mainly exists on an academic level. Some of the research carried out in this field tries to mimic the behaviour of electric buck and boost converters by applying fast switching valves taking advantage of the fluid inertia effects to create oscillator circuits [1], [2]. In other research, inertial effects are added by mechanical means [3], [4], e.g. a series connected hydraulic machine driving a flywheel. Other work instead focus on transformation through the mechanical domain, using either rotary or linear digital controlled machines [5], [6]. The linear transformer has the advantage of only two domain transformations; from hydraulic to linear mechanic and back to hydraulic. The rotary machine normally takes four steps also counting the transformation between linear moving pistons to rotary movement of the shaft and back again. On the other hand, the linear transformer has a finite stroke and requires continuous switching in direction.

The idea of transforming hydraulic energy via the mechanical domain is nothing new. Already in the early 80 's significant research was done on very similar concepts [7], [8], but at that time using conventional variable displacement machines rather than digital hydraulic machi- nes. An interesting challenge here is controllability. Since the machine has a free rotating axle with very low inertia, it is highly sensitive to torque disturbances. To keep a stable operation, good hardware as well as intelligent controls are needed. Due to the finite number of pistons, torque ripple is a problem making low speed operation a challenge. From the late 90's up until today much research was done on alternative transformer principles where one machine may be used instead of two in an endeavour to make the component easier to control, more energy efficient and commercially viable, see [9]. However, less research is found on how modern control theories may be applied to overcome the known hurdles in control of these transformers.

From the hydraulic system point of view the hydraulic transformer may be applied in several different ways. Some examples can be found in [10], [11] and [12]. One way is to use one transformer per function, where each transformer controls the flow to one actuator, see fig. 1. Alternatively, the transformer may be used as a load-sensing pump supplying flow to a load-sensing control valve. Another option is to place the transformer in parallel to a conventional circuit, as shown in fig. 22 This allows energy to be recuperated and stored when possible and energy reused when needed. In this paper, the "parallel" transformer concept is targeted, even though the presented control theories also applies to the other two concepts with minor modifications. The transformer is built up by two variable displacement machines, as previously described in [13]. With standard hydraulic machines, this configuration gives a rather poor round-trip efficiency, which limits its use for energy recuperation. With new technology 
and high-efficient hydraulic machines, however, this may change. Another important contribution of an add-on energy storage system might be that it can allow for downsizing of the engine. This paper analyses the described system dynamically and suggests a control strategy based on linear techniques. A control algorithm is tested and evaluated both in simulation and in hardware tests.

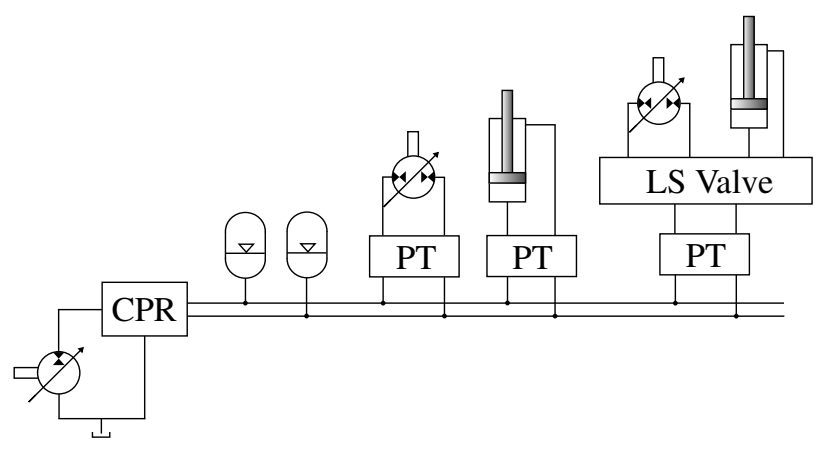

Figure 1: One possible configuration of a transformer based system. A common pressure rail (CPR) supports the applications with pressure. The flow to each actuator and the load sensing (LS) valve control system is controlled by separate pressure transformers $(P T)$.
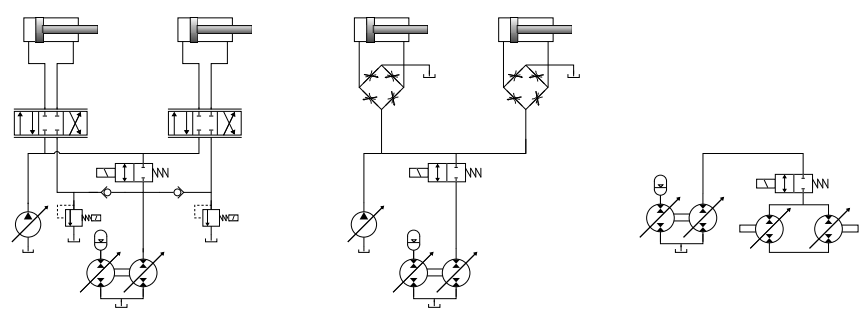

Figure 2: Examples of how the transformer-based system can be connected to standard hydraulic systems in a parallel structure, from [13].

\section{Modelling}

The model of the system is used both in the proposed modelbased controller and also to evaluate the proposed control approach. The model is kept relatively simple to achieve a robust and reliable controller. The suggested level of detail serves this purpose, although additional modelling effort would of course increase the precision. The system is modelled as a rotating mass with inertia $J$, connected to the two displacement machines according to fig. 3. The shaft torque on the transformer is generated from the pressure on the load and accumulator side, $p_{L}$ and $p_{A}$, in reference to the low side pressure $p_{T}$. The relative displacement of the displacement machines are determined by the control signals $\varepsilon_{A}$ and $\varepsilon_{L}$. The losses in flow and torque has been modelled with separate efficiency models $\left(\eta_{A}, \eta_{L}\right)$ for the two displacement machines. The dynamic equations for the system are shown in eq. (1). The net torque (ideal torque minus torque losses) affects the acceleration of the transformers inertial load. The control dynamics of the the displacement machines are modelled with

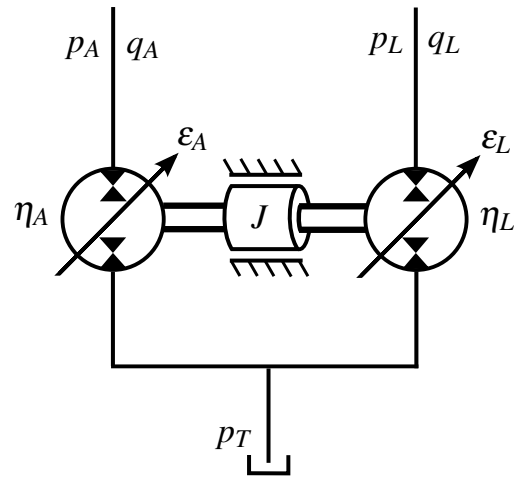

Figure 3: Transformer model.

a first order system with time constants $\tau_{L}$ and $\tau_{A}$. The load side flow is dependent on the rotational speed and the load side displacement.

$$
\begin{aligned}
& J \dot{\omega}=T_{L, \text { ideal }}-T_{A, \text { ideal }}-T_{\text {loss }}(\varepsilon, p, \omega) \\
& \dot{\varepsilon_{L}}=-\frac{\varepsilon_{L}}{\tau_{L}}+\frac{\varepsilon_{L, \text { ref }}}{\tau_{L}} \\
& \dot{\varepsilon_{A}}=-\frac{\varepsilon_{A}}{\tau_{A}}+\frac{\varepsilon_{A, r e f}}{\tau_{A}} \\
& q_{L}=\varepsilon_{L} D_{L} \omega \pm q_{\text {loss }}\left(\varepsilon_{L}, \omega\right)
\end{aligned}
$$

where

$$
\begin{aligned}
& T_{i, \text { ideal }}=\frac{\varepsilon_{i} D_{i}}{2 \pi} \Delta p_{i}, \text { for } i=L, A \\
& \Delta p_{i}=p_{i}-p_{T}, \text { for } i=L, A
\end{aligned}
$$

\subsection{Loss modelling}

The loss modelling of the displacement machines are quite critical to the performance of the controller, since both torque and flow losses are dependent on the surrounding operating conditions. Different modelling approaches have been suggested over the years with different level of complexity and accuracy [14]. Rydberg [15] suggested a physical model of the torque and flow losses of axial piston machines. The proposed models in this paper is based on Rydberg's models, with modifications to reduce the complexity of the expressions.

\subsubsection{Flow losses}

The flow losses on the load side has been modelled with a two parameter model according to eq. (3). The model consists of one constant term and one term depending on the relative displacement. No bias is assumed for zero speeds. This model is able to capture the flow losses in the limited operating area that has been tested in this paper. To increase the quality of the model in a larger operating area, additional terms are necessary.

$$
q_{\text {loss }}=a_{0} \varepsilon_{L} D_{L} \omega+a_{1} \varepsilon_{L}^{2} D_{L} \omega
$$

\subsubsection{Torque losses}

The torque losses for the complete transformer are modelled as a function of relative displacement, pressure and rotational 
speed, according to eq. (4).

$$
\begin{aligned}
T_{\text {loss }}= & \left(b_{0}+b_{1} \varepsilon_{L}\right) \frac{D_{L}}{2 \pi} \Delta p_{L}+\left(c_{0}+c_{1} \varepsilon_{A}\right) \frac{D_{A}}{2 \pi} \Delta p_{A}+ \\
& \left(C_{d 1} \varepsilon_{L}+C_{d 2} \varepsilon_{A}\right) \omega+T_{C}
\end{aligned}
$$

The temperature is also an important parameter affecting the friction of the transformer. To reduce the degrees of freedom, the system is evaluated for a fixed temperature.

\section{Control Approach}

The control strategy for the system is a feed forward link based on the non-linear system model in section 2 . The control signals of the transformer are calculated to get the requested flow, $q_{L, \text { ref }}$ (see fig. 4). To compensate for model errors, the measured and estimated states are used in a feedback link that compensates the control signal. Since the flow cannot be measured directly, it is estimated through an observer that uses the modelled flow losses. Previous research of the system can be found in [13], where a similar control strategy was evaluated for the accumulator side displacement machine, together with a fixed value for the load side displacement.

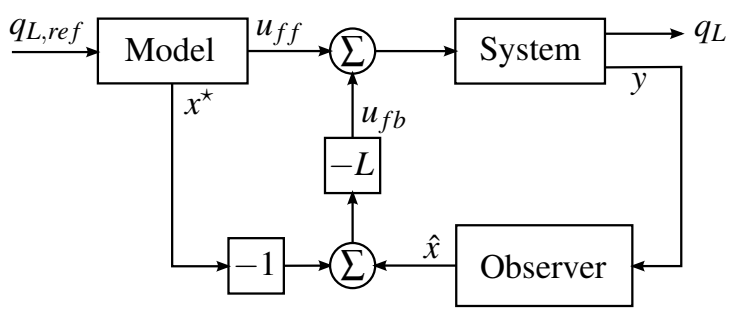

Figure 4: Overview of the control strategy for the system. The model of the system calculates the desired states and inputs. Feedback of state error is used to compensate for model errors. The flow cannot be measured directly and is estimated by an observer.

\subsection{Feed forward}

The feed forward link used two separate control loops to control the relative displacement on each displacement machine (see fig. 5). An inner control loop uses the reference flow to control the relative displacement on the load side, $\varepsilon_{L, f f}$. The goal is to choose a displacement that gives a suitable rotational speed, $\omega_{r e f}$, of the transformer. Due to the limited conversion range of the transformer it is not always possible to run at the requested angular velocity for all reference flows. Based on the load side control signal from the inner control loop, a secondary loop uses the non-linear torque loss model to control the relative displacement on the accumulator side, $\varepsilon_{A, f f}$.

\subsection{Feedback}

To compensate for model errors, the controller uses the measured and estimated states to compensate the control signals

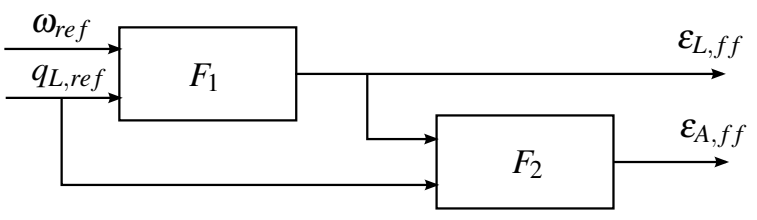

Figure 5: Overview of the control loops for the feed forward link. The control block $F_{1}$ controls the relative displacement on the load side and $F_{2}$ controls the relative displacement on the accumulator side.

on both displacement machines. This is done by applying a feedback gain matrix, L, on the controlled state which results in a control signal that is added as input to the system. The static feedback gain cannot fully compensate for stationary errors. For this reason, an additional, integrated state can be introduced (see [16]). For this system, the integrated state feedback is activated within a finite area around the requested flow so that it will not affect the behaviour during transients in the flow.

\subsection{Extra degree of freedom}

The inner control loop for the load side displacement provides an extra degree of freedom, where the working speed of the transformer can be controlled. Working at a low rotational speed can minimize the torque losses but also compromises the performance since the stick-slip effect are prominent for low speeds. Working at higher speeds reduces these effect and improves the performance, but may lead to larger torque losses. Additional studies of this control aspect can be found in [10] where the transformer speed and load side flow of a two-machine transformer are controlled by separate PI-controllers.

\section{Linear analysis}

To investigate the properties of the system in different parts of its operating area, the non-linear model of the system is linearised in different operating points and analysed. For each operating point, a state space model of the system is created.

$$
\begin{aligned}
& \dot{x}=A x+B u \\
& y=C x
\end{aligned}
$$

where

$$
\begin{aligned}
& x=\left[\Delta q_{L}, \Delta \varepsilon_{L}, \Delta \varepsilon_{A}, \Delta \omega\right]^{T} \\
& u=\left[\Delta \varepsilon_{L, \text { ref }}, \Delta \varepsilon_{A, r e f}\right]^{T}
\end{aligned}
$$

Using the friction model previously described in section 2.1.2 the mathematical equations are used to form the state space matrices. The dynamics of the pressures are assumed to be small enough for the pressures to be treated as constants at each operating point. The performance for different feedback gains has been investigated by forming the state space model of the closed loop system. With feedback, the input to the system is a function of the state, as in eq. (7).

$$
u=-L x
$$


This results in the closed loop system in eq. (8).

$$
\begin{aligned}
& \dot{x}=(A-B L) x \\
& y=C x
\end{aligned}
$$

Depending on the design of the matrix $L$ it is possible to control the impact of each state on the control signals. In this paper, the feedback link is designed to compensate both the load side and the accumulator side displacement based on the estimated flow. By letting both displacement machines be affected instead of just one, the performance is increased.

To find a suitable value for the gain, the pole placement with respect to the feedback gain is analysed. Figure 6 shows the placement of the poles for an increasing feedback gain. The system has four poles. Two of them are placed on the

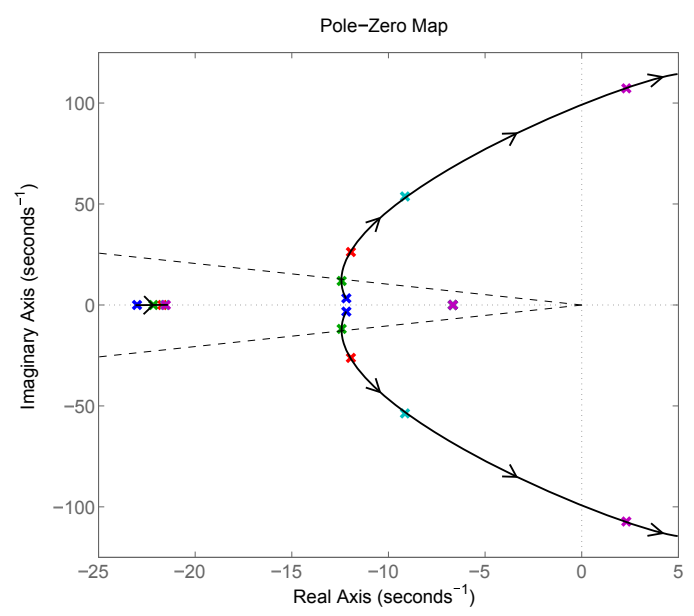

Figure 6: The poles of the closed loop system in a common operating point for different values of feedback gain. The dashed lines indicates the area in which the poles should be placed to avoid large oscillations.

real axis and the other two are complex-conjugated with both a real and an imaginary part. An increased feedback gain pushes the complex-conjugated pair of poles along the positive real axis and increases the imaginary part. This leads to increased oscillations for the system and ultimately instability when the poles reaches the right half plane. The dashed lines represents a damping of 0.7. A common demand is to keep the poles within this area to prevent to large oscillations in the system. It is clear that the poles for the closed loop system are quickly moved out of this area even for small values of feedback gain. Since the load side pressure source on the hardware test bench is based on a flow source and a pressure control valve, it is extra sensitive to oscillations in the system. The oscillations cause pressure disturbances which can lead to instability if they are too large.

\subsection{Variance between operating points}

The sensitivity of feedback gain is different between operating points. Figure 7 and 8 shows the effect of an increased

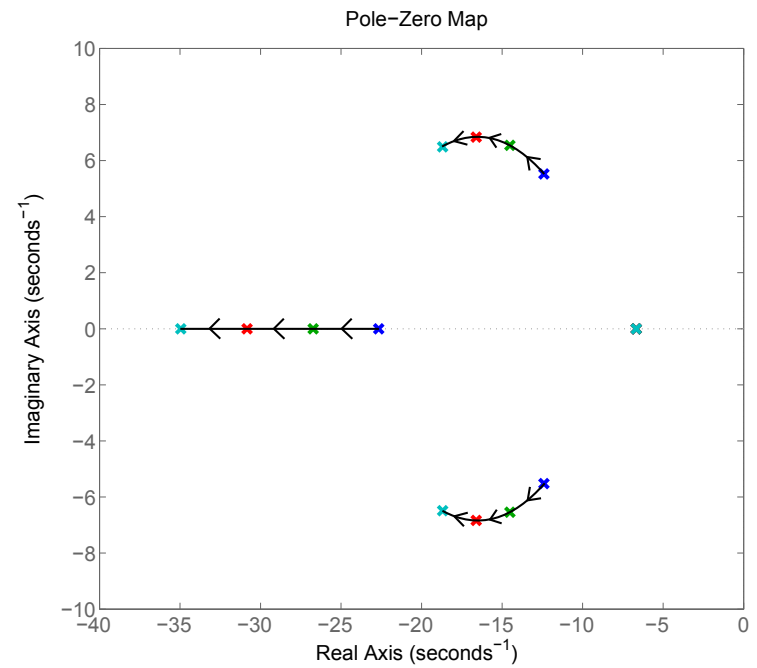

Figure 7: Increasing load pressure.

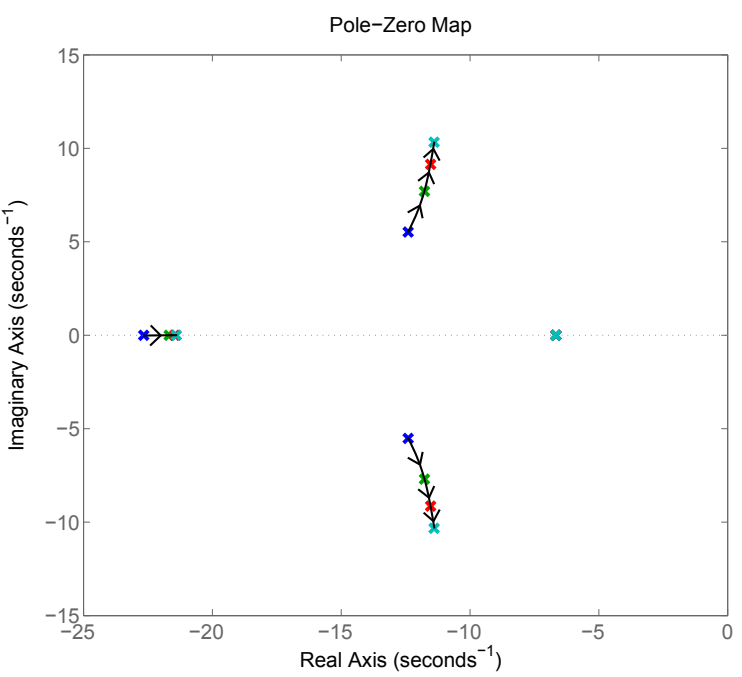

Figure 8: Increasing accumulator pressure.

load and accumulator pressure on the closed loop system. An increased load side pressure moves three of the poles along the negative real axis with a small effect on the imaginary part of the poles. This causes faster dynamics for the system. Increasing accumulator pressure pushes two of the poles along the imaginary axis which leads to larger oscillations in the system. The behaviour of the closed loop system in different operating points can be optimised by changing the feedback gain based on the the current operating point. This will be discussed briefly in section 5

\subsection{Dynamics of the control units}

The performance of the closed loop system is highly dependent on the dynamics of the control units which control the relative displacements of the transformer. The low inertia of the transformer shaft makes the system sensitive to disturbances which immediately impacts the performance. To compensate 
for these disturbances, fast respone time is needed from the displacement machines. Figure 9 and 10 shows the normalized frequency response between the relative displacement on load and accumulator side respectively and the load side flow for different dynamics. The time constants for the swash plate servo is varied from 0.05 to 0.5 seconds. The frequency re-

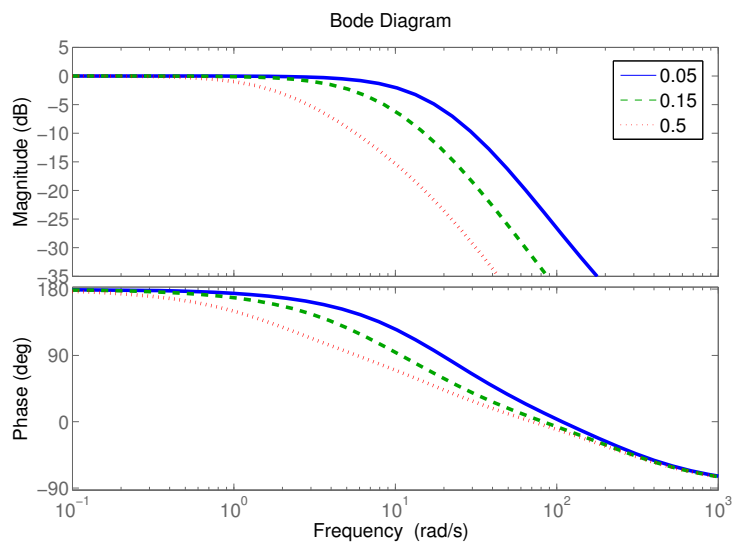

Figure 9: The frequency response between $\varepsilon_{L}$ and $q_{L}$ for different dynamics in the swash plate setting.

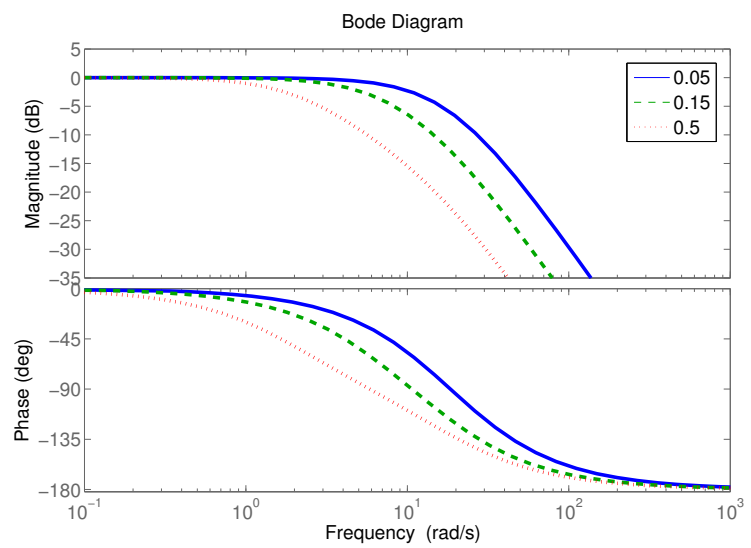

Figure 10: The frequency response between $\varepsilon_{A}$ and $q_{L}$ for different dynamics in the swash plate setting.

sponse from the control signals to the load side flow is directly affected by the dynamics of the control unit. The required response times of the machines are of course coupled to the inertia of the transformer.

\subsection{Dynamics for an increased inertia}

As previously discussed, the small inertia of the system is problematic since it leads to quick changes in the rotational speed while the control dynamics are comparatively slow. For this reason, a study of the pole placement is made for an increasing inertia on the transformer, with the inertia increased 2, 5 and 10 times the original value. This is shown in fig. 11 As expected, an increased inertia decreases the imaginary part of the poles which reduces the oscillations in the system. At the same time, it pushes the poles closer to the origin which leads to slower dynamics. It is clear that while a high inertia

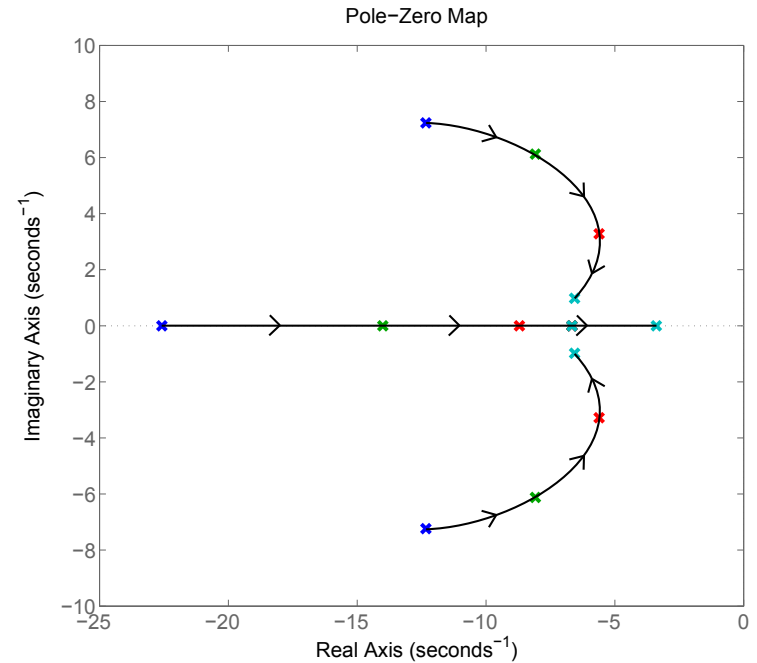

Figure 11: The pole placement of the closed loop system for an increasing inertia. Starting with the original value of $J$ the inertia is then increased 2, 5 and 10 times.

could allow for an increased feedback gain and less sensitivity to disturbances, it will slow down the response time for the system as well.

\section{Gain scheduling}

To compensate for the varying feedback sensitivity between operating points, the feedback gain can be implemented as a function of the variables in the system. This makes it possible to achieve optimal performance for the feedback link in the full operating area of the transformer. One way to accomplish this is to scale the feedback gain matrix $L$, with an operating point dependent function $K_{\text {gain }}$. For this system, the function is chosen as a function of accumulator and load side pressure, according to the analysis in section 4.1

$$
K_{\text {gain }}=f\left(p_{A}, p_{L}\right)
$$

\section{Start-up procedure}

Achieving a requested load flow from zero-speed operation is problematic due to large stick-slip effects on the transformer for low speed operations [17]. Figure 12] shows the magnitude of the friction torque on the transformer as a function of the rotational speed. For zero speed, a large torque is needed to overcome the stiction torque, $T_{s t}$. Once the transformer is in motion, the stick-slip effects still have great influence on the torque losses for lower speeds but are reduced for higher speeds where the Coulomb friction $\left(T_{c}\right)$ and viscous friction is dominating. To compensate for the stiction torque it is necessary to create an overshoot in the torque on the displacement machines, that sets the transformer into motion. Once the transformer is running, the stick-slip effects are reduced and the overshoot torque is no longer necessary. One way to implement this overshoot is to add a term, $u_{\text {start-up }}$, to the original feed forward control signal, $u_{f f 0}$ (see [17]). This results 


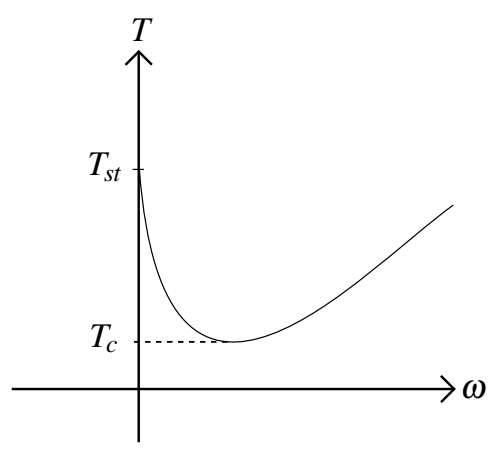

Figure 12: Principle of the stiction torque acting on the transformer. During low speed operations, the transformer is subject to large stick-slip effects. These are reduced for higher speeds.

in the feed forward control signal described by eq. 10.

$$
u_{f f}=u_{\text {start }-u p}+u_{f f 0}
$$

The term $u_{\text {start-up }}$ creates a peak in the control signals while the transformer speed is zero, which rapidly declines after the transformer starts to rotate. For the system described in this paper, such a peak will be used as the control signal on one side of the transformer while the control signal on the other side is set to zero. The peak is generated by eq. 111.

$$
u_{\text {start-up }}= \pm K^{-\frac{\omega}{\omega_{0}}}
$$

The sign of the function is depending on the direction of the requested flow. For zero speed, the control signal is $u_{\text {start }- \text { up }}=-1$, when a negative flow is requested. This value moves quickly towards zero for increasing rotational speeds. The fix value $\omega_{0}$ is a speed where the stick-slip effects are assumed to no longer affect the transformer. At this speed, the control signal is $-\frac{1}{K}$ and declines further towards zero for increasing speeds. Figure 13 shows the output from this function as a function of the rotational speed.

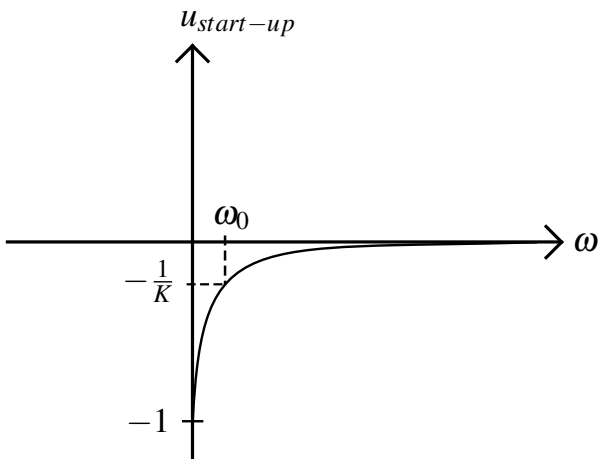

Figure 13: The extra control signal used to set the transformer into motion.

\section{Hardware}

A hardware test bench has previously been developed (see [13] and [18] ) to evaluate the system concept and control strategy. The test bench is shown in fig. 14 and consists of two Bosch Rexroth (A4VG) displacement machines connected via a mechanical shaft. The relative displacement and pressure at each machine is measurable as well as the rotational speed of the shaft. Figure 15 shows the schematic overview of the test bench. Further details can be found in the above referenced documents.

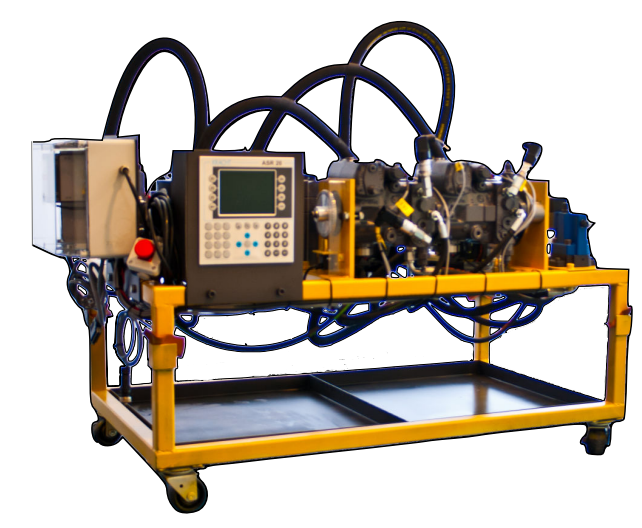

Figure 14: The hardware test bench used to evaluate the controller.

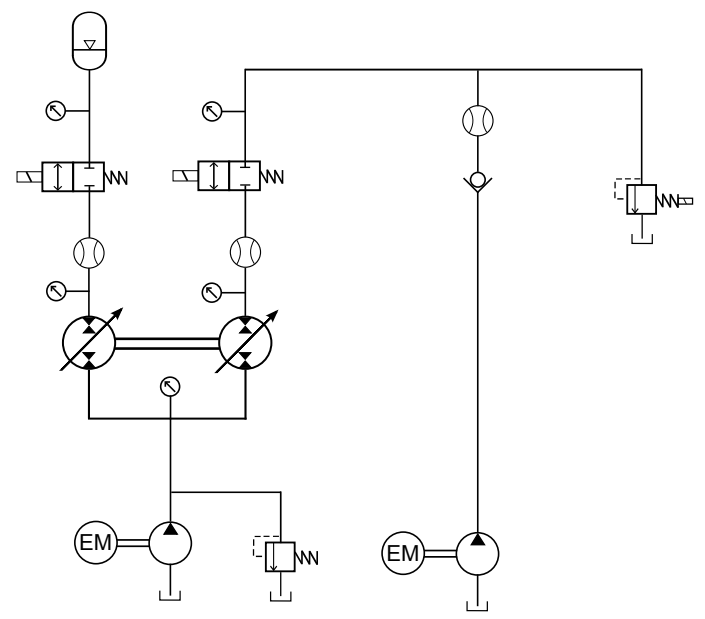

Figure 15: Schematic overview of the test bench. The pressures on the load side and the low-pressure side of the transformer are controlled by two separate pump systems and pressure relief valves. The flow sensors are used for validation and not for controlling.

\section{Results and Discussions}

The performance of the controller has been evaluated on both a simulation model and the hardware test bench. The simulation model is implemented in the modelling software AMESim. The standard library components have been modified according to the above described system model. 
The evaluation is performed through cosimulation between MATLAB Simulink and AMESim [19].

\subsection{Reference tracking}

The controllers ability to follow a reference flow has been evaluated on the simulation model as well as the hardware test bench. Figure 16a shows the simulation results with the corresponding displacement shown in fig. 16b. The control-

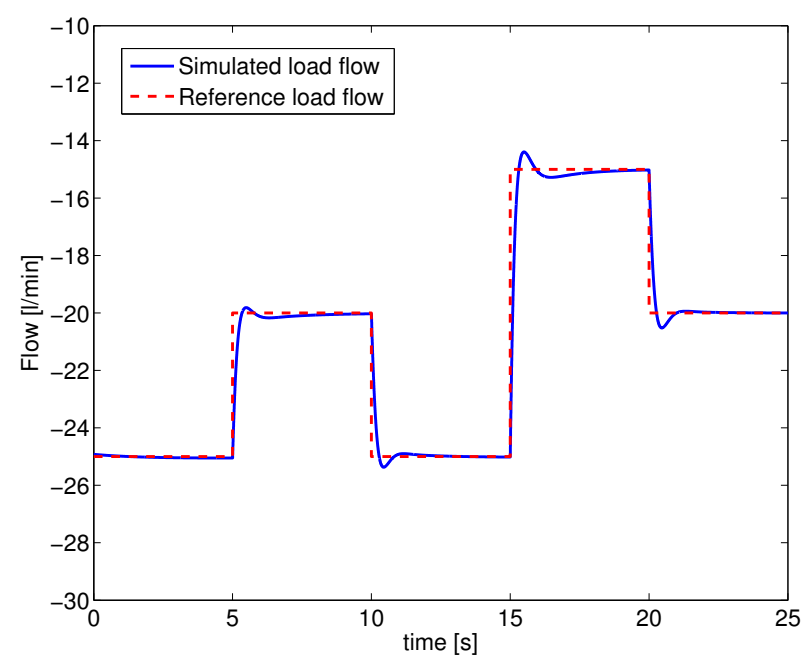

(a) Load side flow.

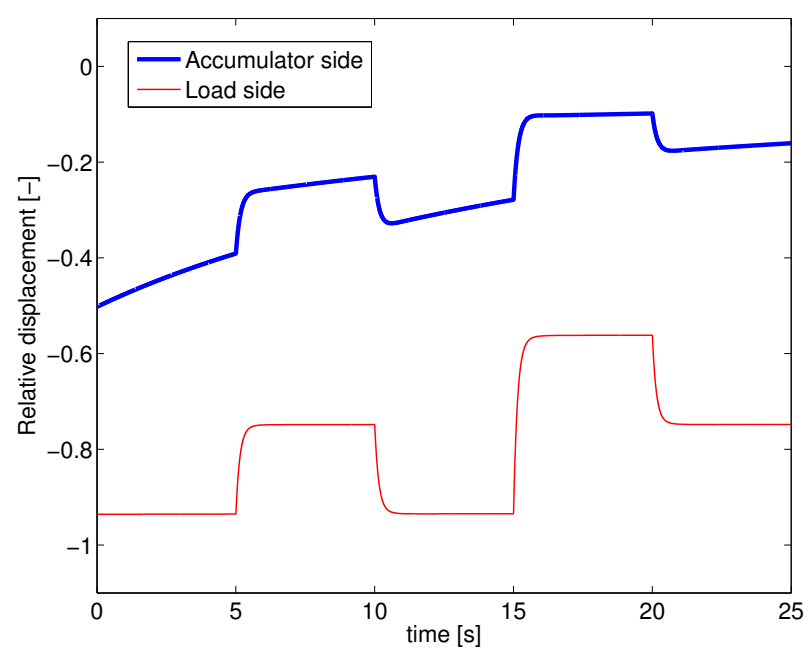

(b) Relative displacement.

Figure 16: Reference tracking experiment on the simulation model.

ler causes both displacement machines to react to steps in the load side flow. The feedback link adjusts the control signal on both sides which provides for superior compensation for model errors. This does, however, lead to a small overshoot in the flow before the stationary value is reached. Figure 17 shows an equivalent evaluation on the hardware test bench. The feed forward link is highly dependent on an accurate model of the torque losses, which are harder to estimate in the hardware experiments. Unmodelled non-linearities and temperature dependency cause stationary errors which are partly compensated by the feedback link.

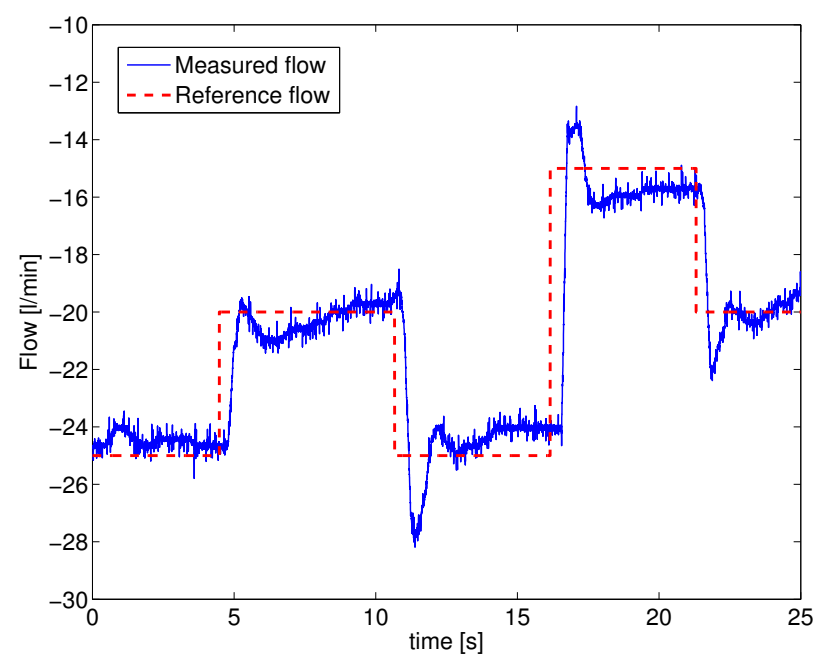

(a) Load side flow.

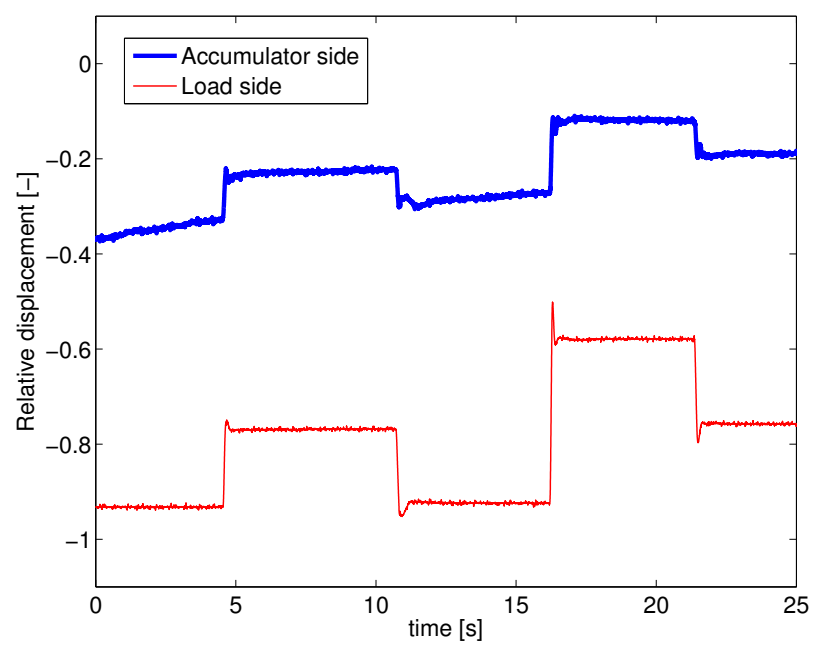

(b) Relative displacement.

Figure 17: Reference tracking experiment on the hardware test bench.

\subsection{Suppression of load disturbances}

Another important aspect of the controller is the ability to compensate for disturbances in the load side pressure. Figure 18 shows a hardware test result of a $10 \%$ step in load side pressure in each direction. The feed forward link is able to compensate for the pressure disturbances by adjusting the relative displacement on the accumulator side. However, the relatively slow dynamics of the displacement machines cause an unsatisfactory high flow disturbance. In a real application, e.g. with the transformer connected to a hydraulic crane, the force from the lift load would create pressure oscillations that need to be suppressed. The problem with slow displacement 


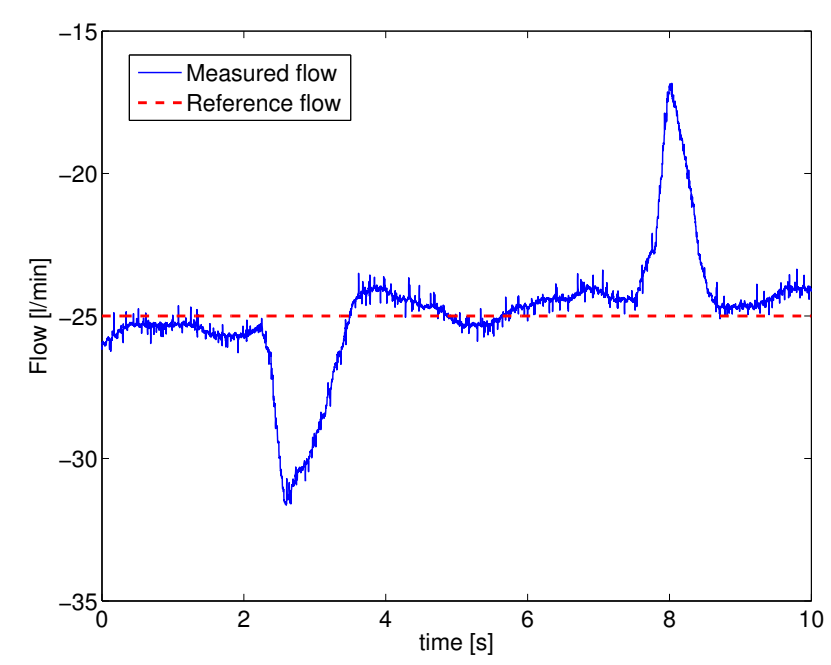

(a) Load side flow.

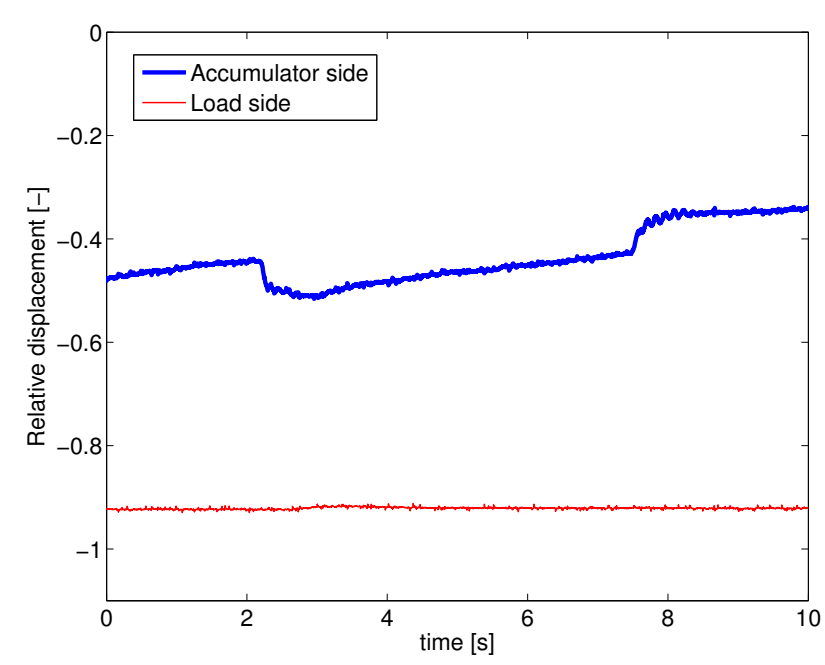

(b) Relative displacement.

Figure 18: Hardware experiment where a step in the load side pressure of $10 \%$ is made in each direction.

machines is also treated in [10], where a crane arm is controlled by a similar system.

\subsection{Start-up procedure}

An evaluation of the start-up procedure on the test bench is shown in fig. 19. Both the reference and actual flow is presented, as well as the relative displacement on each side. A step in reference flow is made from $0 \mathrm{l} / \mathrm{min}$ to $-15 \mathrm{l} / \mathrm{min}$ at a point where the transformer speed is zero. To overcome the stiction torque, an overshoot in the relative displacement is given until the transformer starts to rotate. This leads to an overshoot in the load flow before the flow is controlled to the requested value. The transformer shaft is accelerated to a high speed before the system is able to react and adjust the control signal. Certain delays in the measurement equipment also contributes to the magnitude of the overshoot.

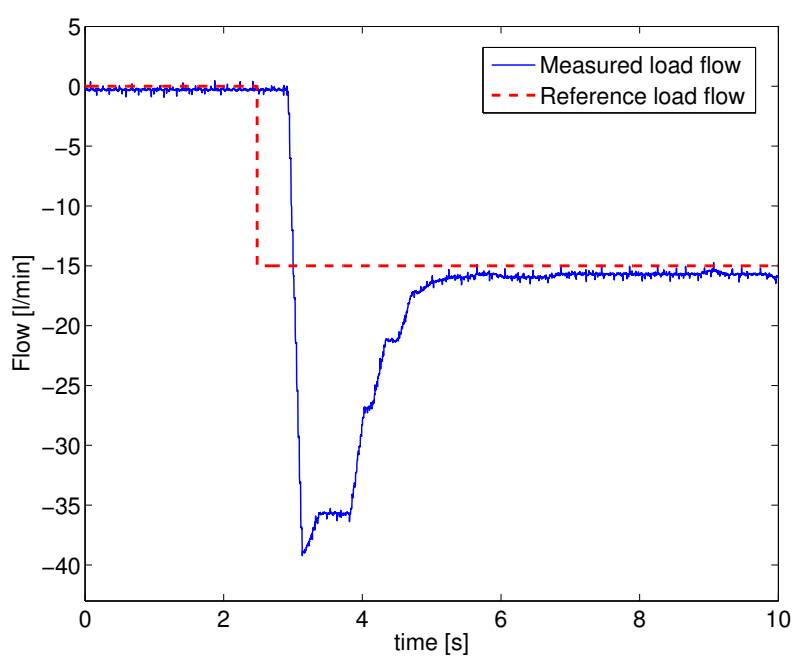

(a) Load side flow.

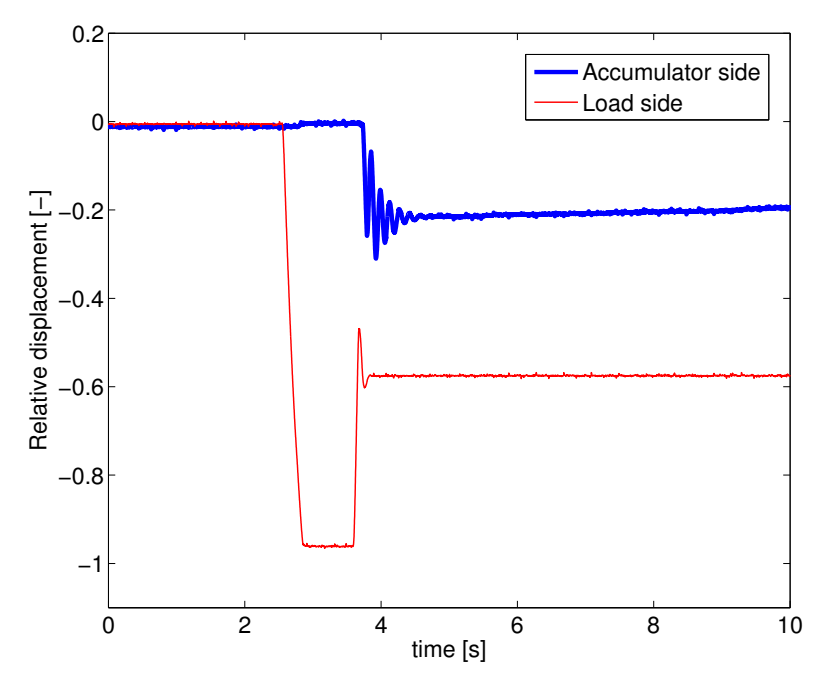

(b) Relative displacement.

Figure 19: Evaluation of the start-up procedure on the hardware test bench.

\section{Conclusion}

A control strategy for a transformer-based energy recuperation system has been proposed and evaluated. The control strategy is based on a non-linear feed forward link and a state feedback link that compensates for model-errors and disturbances. The implementation of separate control loops for each displacement machine reduces the control problem to a single input single output system. This simplifies the control algorithm and provides the possibility to affect the working speed of the transformer as well. The model based feed forward control signal allows for good reference tracking, but it is highly dependent on correct estimations of the torque losses. Due to strong non-linearities in the friction between operating points, this is a major issue in the control problem. Another issue is the small inertia and slow control dynamics, which cause challenges for the start-up process 
and difficulties when compensating for disturbances.

The fundamental properties of the system has been investigated through linear methods. The problem with low inertia and slow control dynamics is visualised by the frequency response and the pole placement of the closed loop system. The ability to react to changes in the load flow is directly affected by the response time for the control units. The small margin for feedback gain is another consequence, where oscillations and instability follows an increasing feedback gain.

\section{Nomenclature}

\section{Quantities}

\begin{tabular}{lll}
\hline Designation & Denotation & Unit \\
\hline$p$ & Pressure & {$[\mathrm{Pa}]$} \\
$q$ & Flow & {$\left[\mathrm{m}^{3} / \mathrm{s}\right]$} \\
$\varepsilon$ & Relative displacement & {$[-]$} \\
$\omega$ & Rotational velocity & {$[\mathrm{rev} / \mathrm{s}]$} \\
$J$ & Moment of inertia & {$\left[\mathrm{kgm}^{2}\right]$} \\
$T$ & Torque & {$[\mathrm{Nm}]$} \\
$T_{C}$ & Coulomb torque & {$[\mathrm{Nm}]$} \\
$T_{s t}$ & Stiction torque & {$[\mathrm{Nm}]$} \\
$\eta$ & Efficiency & {$[-]$} \\
$\tau$ & Time constant & {$[\mathrm{s}]$} \\
\hline
\end{tabular}

\section{Subscripts}

\begin{tabular}{ll}
\hline Designation & Denotation \\
\hline$T$ & Low pressure (Tank) side \\
$L$ & Load side \\
$A$ & Accumulator side \\
loss & Machine losses \\
$r e f$ & Reference value \\
$f f$ & Feed forward \\
$f b$ & Feedback \\
\hline
\end{tabular}

\section{References}

[1] E. Guglielmino, C. Semini, H. Kogler, R. Scheidl, and D. G. Caldwell, "Power Hydraulics - Switched Mode Control of Hydraulic Actuation," in The 2010 IEEE/RSJ International Conference on Intelligent Robots and Systems, pp. 3031-3036, 2010.

[2] R. Scheidl, B. Manhartsgruber, and B. Winkler, "Hydraulic Switching Control - Principles and State of the Art," in 1st Workshop on Digital Fluid Power, (Tampere, Finland), pp. 31-49, 2008.

[3] F. Wang, L. Gu, and Y. Chen, "A Continuously Variable Hydraulic Pressure Converter Based on High Speed On-Off Valves," Mechatronics, vol. 21, no. 8, pp. 12981308, 2011.
[4] M. B. Rannow, H. C. Tu, P. Y. Li, and T. R. Chase, "Software Enabled Virtually Variable Displacement Pumps - Theoretical and Experimental Studies," ASME Journal of Dynamic Systems , Measurement, and Control, no. April, 2007.

[5] M. Linjama and J. Tammisto, "New Alternative for Digital Pump-Motor-Transformer," in The Second Workshop on Digital Fluid Power, (Linz, Austria), pp. 49-61, 2009.

[6] E. D. Bishop, "Digital Hydraulic Transformer - Approaching Theoretical Perfection in Hydraulic Drive Efficiency," in The 11th Scandinavian International Conference on Fluid Power, (Linköping, Sweden), 2009.

[7] M. Shih, Untersuchung Einer Zylinderansteuerung durch Hydro-Transformator am Konstant Drucknetz. $\mathrm{PhD}$ thesis, Rheinisch-Westfälischen Technischen HochSchule Aachen, 1984.

[8] R. Kordak, Hydrostatische Antriebe mit Sekundärregelung. 1996.

[9] P. A. J. Achten, Z. Fu, and G. E. M. Vael, “Transforming future hydraulics : a new design of a hydraulic transformer," in 5th Scandinavian International Conference on Fluid Power, (Linköping, Sweden), pp. 1-24, 1997.

[10] S. Wei, On Studies of Energy Regeneration System for Hydraulic Manipulators. PhD thesis, Tampere University of Technology, 2004.

[11] P. A. J. Achten, G. E. M. Vael, H. Murrenhoff, T. Kohmäscher, and M. Inderelst, "Low-emission Hydraulic Hybrid for Passenger Cars," ATZ, vol. 5, pp. 378-384, 2009.

[12] M. Inderelst, "Energy Efficient System Layout for Work Hydraulics of Excavators," in The 12th Scandinavian International Conference on Fluid Power, (Tampere, Finland), 2011.

[13] K. Pettersson, K. Heybroek, A. Klintemyr, and P. Krus, "Analysis and control of a complementary energy recuperation system," in The 8th International Fluiid Power Conference, (Dresden, Germany), 2012.

[14] T. Kohmäscher, "Improved Loss Modeling of Hydrostatic Units - Requirement for Precise Simulation of Mobile Working Machine Drivelines," in ASME International Mechanical Engineering Congress and Exposition, Seattle, Washington, USA, vol. 4, (Seattle, Washington), 2007.

[15] K.-E. Rydberg, On Performance Optimization and Digital Control of Hydrostatic Drives for Vehicle Applications. PhD thesis, Linköping University, 1983.

[16] J. Lennevi, Hydrostatic Transmission Control. PhD thesis, Linköping University, 1995. 
[17] R. Werndin and J. Palmberg, "Hydraulic Transformers in Low Speed Operation - A Study of Control Strategies," in The 5th International Symposium of Fluid Power, (Nara, Japan), 2002.

[18] A. Klintemyr and R. Bergström, "Analysis and Sizing of a System for Hydraulic Energy Recovery," Master's thesis, Linköping Univeristy, Linköping, 2011.

[19] LMS International, "LMS Imagine.Lab AMESim Rev 11 User Manual and Software." 